\title{
Adverse Events Due to Insomnia Drugs Reported in a Regulatory Database and Online Patient Reviews: Comparative Study
}

Jill S Borchert ${ }^{1}$, PharmD; Bo Wang ${ }^{2}$, BS; Muzaina Ramzanali ${ }^{1}$, PharmD; Amy B Stein ${ }^{3}$, PhD; Latha M Malaiyandi ${ }^{4}$, $\mathrm{PhD}$; Kirk E Dineley ${ }^{4} \mathrm{PhD}$

\footnotetext{
${ }^{1}$ Chicago College of Pharmacy, Midwestern University, Downers Grove, IL, United States

${ }^{2}$ Chicago College of Osteopathic Medicine, Midwestern University, Downers Grove, IL, United States

${ }^{3}$ Office of Research and Sponsored Programs, Midwestern University, Glendale, AZ, United States

${ }^{4}$ College of Graduate Studies, Midwestern University, Downers Grove, IL, United States
}

Corresponding Author:

Kirk E Dineley, PhD

College of Graduate Studies

Midwestern University

555 31st Street

Downers Grove, IL, 60515

United States

Phone: 16309603907

Email: kdinel@midwestern.edu

\section{Abstract}

Background: Patient online drug reviews are a resource for other patients seeking information about the practical benefits and drawbacks of drug therapies. Patient reviews may also serve as a source of postmarketing safety data that are more user-friendly than regulatory databases. However, the reliability of online reviews has been questioned, because they do not undergo professional review and lack means of verification.

Objective: We evaluated online reviews of hypnotic medications, because they are commonly used and their therapeutic efficacy is particularly amenable to patient self-evaluation. Our primary objective was to compare the types and frequencies of adverse events reported to the Food and Drug Administration Adverse Event Reporting System (FAERS) with analogous information in patient reviews on the consumer health website Drugs.com. The secondary objectives were to describe patient reports of efficacy and adverse events and assess the influence of medication cost, effectiveness, and adverse events on user ratings of hypnotic medications.

Methods: Patient ratings and narratives were retrieved from 1407 reviews on Drugs.com between February 2007 and March 2018 for eszopiclone, ramelteon, suvorexant, zaleplon, and zolpidem. Reviews were coded to preferred terms in the Medical Dictionary for Regulatory Activities. These reviews were compared to 5916 cases in the FAERS database from January 2015 to September 2017.

Results: Similar adverse events were reported to both Drugs.com and FAERS. Both resources identified a lack of efficacy as a common complaint for all five drugs. Both resources revealed that amnesia commonly occurs with eszopiclone, zaleplon, and zolpidem, while nightmares commonly occur with suvorexant. Compared to FAERS, online reviews of zolpidem reported a much higher frequency of amnesia and partial sleep activities. User ratings were highest for zolpidem and lowest for suvorexant. Statistical analyses showed that patient ratings are influenced by considerations of efficacy and adverse events, while drug cost is unimportant.

Conclusions: For hypnotic medications, online patient reviews and FAERS emphasized similar adverse events. Online reviewers rated drugs based on perception of efficacy and adverse events. We conclude that online patient reviews of hypnotics are a valid source that can supplement traditional adverse event reporting systems.

(J Med Internet Res 2019;21(11):e13371) doi: 10.2196/13371

\section{KEYWORDS}

drug safety; drug ineffective; postmarketing; pharmacovigilance; internet; pharmacoepidemiology; adverse effect; hypnotic; insomnia; patient-reported outcomes 


\section{Introduction}

Postmarketing surveillance is coordinated by regulatory bodies that use passive collection systems to monitor the occurrence of drug toxicities. The Food and Drug Administration (FDA) Adverse Event Reporting System (FAERS) relies on drug manufacturers, health care professionals, and the patients themselves to report instances of adverse events in the form of an Individual Case Safety Report [1,2]. Although manufacturers are obligated to report adverse events, reporting by health care professionals and patients is voluntary. Consequently, a large amount of safety data never reach regulators [3-5], which underscores the need for additional mechanisms of pharmacovigilance.

Over the past decade, a rapidly expanding body of research has focused on the abundance of health data generated by patient online activities [6-10]. These include data collected from Web browser search habits [11,12], commentary that appears on social media such as Facebook and Twitter [13-16], and information revealed on internet forums $[17,18]$. The field of inquiry concerned with mining these resources for the purposes of improving public health has been referred to as "infodemiology" [19].

One type of Web datum enriched with drug safety information appears on websites such as WebMD, AskaPatient, and Drugs.com in the form of patient drug reviews. In a few sentences or a short paragraph, the patient shares his/her personal experience with efficacy, adverse events, and other issues related to the use of a given drug. Researchers have begun to explore how these reviews might improve pharmacovigilance. For example, online reviews have been used to show that emotional and behavioral effects are prominent considerations for users of antidepressants and antipsychotics [20,21]. Others found that online reviewers tend to describe less serious adverse events than those described by FAERS reports [22]. Web reviews can also help assess illicit drugs that fall outside of conventional trial evaluation [23], although evidence suggests that online commentary tends to minimize the dangers of habit-forming drugs [24]. Taken together, these studies suggest that online patient drug reviews can enhance traditional pharmacovigilance mechanisms by offering quick collection of information from large, diverse populations, followed by rapid dissemination in a form that is accessible to the lay community.

This study compares information contributed by patients to an online drug information website with adverse event data collected by the FDA. We focused on a particular set of FDA-approved hypnotics because they are common drugs used almost exclusively for the treatment of sleep disorders $[25,26]$. This minimized concerns over variable patient experience that arises when the same drugs are used for different therapeutic applications. In addition, hypnotics are well suited to patient evaluation, because the goal of therapy is simple, and most unwanted effects are easily identified. In our primary objective, we compared the frequencies of adverse events reported in FAERS with analogous information that appeared in patient reviews on the website Drugs.com. Secondary objectives were to describe patient reports of efficacy and adverse events and determine whether cost, effectiveness, or adverse events influenced user ratings of hypnotic medications.

\section{Methods}

\section{Hypnotics}

Five hypnotics were selected for this study. Eszopiclone, zaleplon, and zolpidem are a class of benzodiazepine receptor agonist known as Z-drugs and are the most commonly prescribed class of hypnotics [27]. Ramelteon is a melatonin receptor agonist that promotes sleep via activation of the melatonin 1 receptor subtype (MT1) [28]. Suvorexant promotes sleep by blocking OX1 and OX2 orexin receptors and is the first dual orexin receptor antagonist (DORA) approved for clinical use [29]. Other drugs used for insomnia (eg, benzodiazepines, trazodone, antihistamines, and melatonin) were excluded because those agents are frequently used for indications unrelated to sleep disorder or they are not FDA approved for the treatment of sleep disorders.

\section{Online Reviews}

A total of 1407 publicly available online drug reviews concerning either eszopiclone $(n=239)$, ramelteon $(n=72)$, suvorexant $(n=324)$, zaleplon $(n=82)$, or zolpidem $(n=690)$, dated from February 2007 to March 2018, were retrieved from the website Drugs.com, a drug information platform for consumers and health care professionals. Drugs.com allows users to summarize their overall drug experience via anonymous text narratives and a numerical rating system, with 1 indicating not effective and 10 indicating most effective [30]. The total number of online reviews for a given drug is usually greater than the number of numerical ratings for that drug because not all reviewers chose to contribute a numerical summary rating.

Text narratives and numerical ratings from patient reviews were imported into Microsoft Excel (Microsoft Corporation, Redmond, Washington) for evaluation and analysis. A primary coder read each review to identify language used by the patient to convey an adverse event. Those keywords and phrases were then used to manually select low-level terms (LLTs) within MedDRA (Medical Dictionary for Regulatory Activities), version 18.0 [31]. MedDRA terminology is the international medical terminology developed under the auspices of the International Council for Harmonisation of Technical Requirements for Pharmaceuticals for Human Use (ICH). The corresponding preferred terms (PTs) were dictated by the LLT choice, consistent with MedDRA "Points to Consider" guidelines. Each review was also coded for mention of complaints of drug cost or insurance coverage. Microsoft Excel was then used to randomly select a subset of 166 cases that included at least $10 \%$ of the reviews for each of the five drugs. This subset was recoded by two secondary coders working independently and blinded to the LLT and PT selections of the primary coder. The percentage of reviews coded identically between the primary and the secondary coders was $74.7 \%$ and $78.3 \%$, respectively. All three coders then met in person to discuss several recurring themes of disagreement apparent in the subset. For example, some reviews included the term "tolerance" to describe a drug effect that was diminished or lost with continued use ("I developed tolerance" or "I gained 
tolerance"). This was variably coded with the LLT drug effect decreased or drug tolerance increased. The coders concluded that a clinician best assesses tolerance because it is an advanced concept that may include different pharmacokinetic, pharmacodynamic, and behavioral dimensions. In contrast, the patient is often best positioned to determine whether a hypnotic is still working or if the effect has waned. Therefore, narratives that mentioned tolerance were recoded as the LLT drug effect decreased. Reviews mentioning "depression" or "feeling depressed" were similarly problematic. The coders decided that the LLT depression refers to a formal, clinical diagnosis describing a set of symptoms persisting for a minimal length of time and that the mood changes implied in such narratives were more appropriately captured with the LLT depressed mood. Adjustments based on these and other term selections improved primary-secondary coder PT agreement to $79.6 \%$ and $87.3 \%$ of cases, within the subset. Those coding adjustments were then implemented across the entire data set. A small number of reviews mentioned or implied recent or concomitant use of other drugs, but we did not attempt to adjust for that in our data because those instances were rare and difficult to interpret. The primary and secondary coders completed MedDRA training workshops and webinars.

\section{Food and Drug Administration Adverse Event Reporting System}

For FAERS data, 11 quarterly reports (2015-Q1 through 2017-Q3) were downloaded from the FDA website [32]. Microsoft Access (Microsoft Corporation) was used to select 49,389 reports for eszopiclone, ramelteon, suvorexant, zaleplon, or zolpidem based on the "product_ai" field of the DRUG file. To limit the size and complexity of the data set, all reports with multiple drugs were excluded by eliminating cases with more than one "primaryid" entry; subsequently, only reports designating one of the five hypnotics as the primary suspect in the "role_cod" field were selected. These were matched back to the REAC, DEMO, and INDI files to retrieve the adverse event PTs, indications for therapy and demographics. For cases that appeared in multiple quarters due to report updating, we utilized the most recent version. This produced 5916 unique reports concerning either eszopiclone $(n=196)$, ramelteon $(n=103)$, suvorexant $(n=4095)$, zaleplon $(n=37)$, and zolpidem $(n=1485)$ as the only drug reported. These were tabulated in Microsoft Excel for further analysis.

\section{Statistical Analysis}

Statistical analyses were performed using R statistical software (version 3.5.1; R Core Team, Vienna, Austria) using a two-sided significance level of .05. Statistical comparison of user drug ratings was performed using the nonparametric Kruskal-Wallis test, followed by the Dunn posthoc for all pairwise comparisons. To determine the relationship between user rating and complaints about efficacy or cost in the narrative, univariate logistic regression models were fit for each of the drugs separately. Logistic regression coefficients were reported as odds ratios with $95 \%$ CIs. The count of distinct adverse events recorded from the reviews (not including the PTs drug ineffective and drug effect incomplete) was analyzed using Poisson regression, with user rating as the explanatory variable. The exponential of the Poisson regression model coefficients was reported as the incidence rate estimates, and robust standard errors were calculated using the Delta method to control for mild violation of the distribution assumption that the variance equals the mean.

\section{Ethics}

The Institutional Review Board at Midwestern University Downers Grove declared that this project does not qualify as human subjects research. The annotated datasets generated and analyzed during this study are available from the corresponding author on reasonable request.

\section{Results}

\section{Adverse Event Reporting in Online Reviews Versus Food and Drug Administration Adverse Event Reporting System}

The 10 most common MedDRA PT adverse events coded from online patient reviews are shown in Tables 1 and 2, expressed as a percentage of the total number of PTs recorded from all the reviews for each hypnotic. Drug ineffective was commonly reported for all five drugs (eszopiclone: 45/319, 14.1\%; ramelteon: 24/104, 23.1\%; suvorexant: 159/567, 28.0\%; zaleplon: 22/82, 27\%; and zolpidem: 33/958, 3.4\%). Furthermore, partial or limited efficacy was noted in complaints that were coded as drug effect incomplete for ramelteon (12/104, $11.5 \%)$ and suvorexant $(22 / 567,3.9 \%)$. Amnesia was among the top 10 complaints for all three Z-drugs (eszopiclone: 8/319, $2.5 \%$; zaleplon: 4/82, 5\%; zolpidem: $161 / 958,16.8 \%$ ). Certain adverse events are notable for each of the different drugs. Zolpidem was frequently associated with complex partial sleep behaviors, including abnormal sleep-related event (90/958, 9.4\%), sleep-related eating disorder (59/958, 6.2\%), somnambulism (47/958, 4.9\%), and sleep talking (34/958, 3.6\%). Eszopiclone was commonly associated with dysgeusia $(94 / 319$, $29.5 \%)$. Suvorexant reviewers reported distressing parasomnias that included nightmare $(54 / 567,9.5 \%)$, sleep paralysis $(26 / 567$, $4.6 \%)$, and abnormal dreams (25/567, 4.4\%).

Tables 3 and 4 show the top 10 most common PTs in FAERS reports, expressed as the percentage of the total number of PTs for each drug. Drug ineffective emerged as the most common PT recorded in FAERS for each of the five drugs (eszopiclone: 78/458, 17.0\%; ramelteon: 30/208, 14.4\%; suvorexant: 1108/6171, 18.0\%; zaleplon: 18/77, 23\%; zolpidem: 499/3448, $14.5 \%)$. Other PTs common for the individual drugs include amnesia $(51 / 3448,1.5 \%)$ and somnambulism $(75 / 3448,2.2 \%)$ with zolpidem; dysgeusia $(22 / 458,4.8 \%)$ and product substitution issue $(30 / 458,6.6 \%)$ with eszopiclone; and nightmare (422/6171, 6.8\%), abnormal dreams (382/6171, $6.2 \%)$, and sleep paralysis (124/6171, 2.0\%) with suvorexant. 
Table 1. Preferred terms manually coded from online patient reviews of Z-drugs.

\begin{tabular}{|c|c|c|c|c|c|c|}
\hline \multirow[t]{2}{*}{$\#$} & \multicolumn{2}{|l|}{ Eszopiclone $(\mathrm{n}=319)$} & \multicolumn{2}{|l|}{ Zaleplon (n=82) } & \multicolumn{2}{|l|}{ Zolpidem $(\mathrm{n}=958)$} \\
\hline & Preferred term & $\mathrm{n}(\%)$ & Preferred term & $\mathrm{n}(\%)$ & Preferred term & $\mathrm{n}(\%)$ \\
\hline 1 & Dysgeusia & $94(29.5)$ & Drug ineffective & $22(27)$ & Amnesia & $161(16.8)$ \\
\hline 2 & Drug ineffective & $45(14.1)$ & Insomnia & $5(6)$ & Abnormal sleep-related event & $90(9.4)$ \\
\hline 3 & Drug effect decreased & $17(5.3)$ & Somnolence & $5(6)$ & Sleep-related eating disorder & $59(6.2)$ \\
\hline 4 & Product substitution issue & $17(5.3)$ & Amnesia & $4(5)$ & Drug effect decreased & $51(5.3)$ \\
\hline 5 & Insomnia & $10(3.1)$ & Abnormal sleep-related event & $3(4)$ & Somnambulism & $47(4.9)$ \\
\hline 6 & Depressed mood & $9(2.8)$ & Anxiety & $3(4)$ & Sleep talking & $34(3.6)$ \\
\hline 7 & Somnolence & $9(2.8)$ & Hallucination & $3(4)$ & Drug ineffective & $33(3.4)$ \\
\hline 8 & Amnesia & $8(2.5)$ & Headache & $3(4)$ & Drug dependence & $32(3.3)$ \\
\hline 9 & Anxiety & $7(2.2)$ & Restless legs syndrome & $3(4)$ & Somnolence & $28(2.9)$ \\
\hline 10 & Headache & $6(1.9)$ & Abdominal pain upper & $2(2)$ & Hallucination & $27(2.8)$ \\
\hline
\end{tabular}

Table 2. Preferred terms manually coded from online patient reviews of ramelteon and suvorexant.

\begin{tabular}{|c|c|c|c|c|}
\hline \multirow[t]{2}{*}{$\#$} & \multicolumn{2}{|l|}{ Ramelteon $(n=104)$} & \multicolumn{2}{|l|}{ Suvorexant $(\mathrm{n}=567)$} \\
\hline & Preferred term & $\mathrm{n}(\%)$ & Preferred term & $\mathrm{n}(\%)$ \\
\hline 1 & Drug ineffective & $24(23.1)$ & Drug ineffective & $159(28.0)$ \\
\hline 2 & Drug effect incomplete & $12(11.5)$ & Nightmare & $54(9.5)$ \\
\hline 3 & Somnolence & $7(6.7)$ & Headache & $27(4.8)$ \\
\hline 4 & Dizziness & $5(4.8)$ & Somnolence & $27(4.8)$ \\
\hline 5 & Insomnia & $5(4.8)$ & Sleep paralysis & $26(4.6)$ \\
\hline 6 & Abnormal dreams & $4(3.9)$ & Abnormal dreams & $25(4.4)$ \\
\hline 7 & Depressed mood & $4(3.9)$ & Drug effect incomplete & $22(3.9)$ \\
\hline 8 & Feeling abnormal & $4(3.9)$ & Feeling abnormal & $20(3.5)$ \\
\hline 9 & Anxiety & $3(2.9)$ & Insomnia & $19(3.4)$ \\
\hline 10 & Headache & $3(2.9)$ & Hangover & $17(3.0)$ \\
\hline
\end{tabular}

Table 3. Preferred terms retrieved from FAERS reports for Z-drugs.

\begin{tabular}{|c|c|c|c|c|c|c|}
\hline \multirow[t]{2}{*}{ \# } & \multicolumn{2}{|l|}{ Eszopiclone (n=458) } & \multicolumn{2}{|l|}{ Zaleplon (n=77) } & \multicolumn{2}{|l|}{ Zolpidem (n=3448) } \\
\hline & Preferred term & $\mathrm{n}(\%)$ & Preferred term & $\mathrm{n}(\%)$ & Preferred term & $\mathrm{n}(\%)$ \\
\hline 1 & Drug ineffective & $78(17.0)$ & Drug ineffective & $18(23)$ & Drug ineffective & $499(14.5)$ \\
\hline 2 & Insomnia & $33(7.2)$ & Drug effect incomplete & $3(4)$ & Insomnia & $146(4.2)$ \\
\hline 3 & Product substitution issue & $30(6.6)$ & Insomnia & $3(4)$ & Product substitution issue & $96(2.8)$ \\
\hline 4 & Dysgeusia & $22(4.8)$ & Product quality issue & $3(4)$ & Somnambulism & $75(2.2)$ \\
\hline 5 & Product quality issue & $16(3.5)$ & Product substitution issue & $3(4)$ & Somnolence & $59(1.7)$ \\
\hline 6 & Drug effect decreased & $14(3.1)$ & Abnormal behavior & $2(3)$ & Drug dependence & $57(1.7)$ \\
\hline 7 & Delirium & $12(2.6)$ & Drug hypersensitivity & $2(3)$ & Amnesia & $51(1.5)$ \\
\hline 8 & Headache & $7(1.5)$ & Malaise & $2(3)$ & Road traffic accident & $48(1.4)$ \\
\hline 9 & Nausea & $7(1.5)$ & Nausea & $2(3)$ & Toxicity to various agents & $47(1.4)$ \\
\hline 10 & Sleep disorder & $7(1.5)$ & Nightmare & $2(3)$ & Overdose & $46(1.3)$ \\
\hline
\end{tabular}


Table 4. Preferred terms retrieved from FAERS reports for ramelteon and suvorexant.

\begin{tabular}{|c|c|c|c|c|}
\hline \multirow[t]{2}{*}{ \# } & \multicolumn{2}{|l|}{ Ramelteon (n=208) } & \multicolumn{2}{|c|}{ Suvorexant $(\mathrm{n}=6171)$} \\
\hline & Preferred term & $\mathrm{n}(\%)$ & Preferred term & $\mathrm{n}(\%)$ \\
\hline 1 & Drug ineffective & $30(14.4)$ & Drug ineffective & $1108(18.0)$ \\
\hline 2 & No adverse event & $17(8.2)$ & Nightmare & $422(6.8)$ \\
\hline 3 & Intentional overdose & $14(6.7)$ & Abnormal dreams & $382(6.2)$ \\
\hline 4 & Toxicity to various agents & $13(6.3)$ & Somnolence & $256(4.2)$ \\
\hline 5 & Somnolence & $8(3.9)$ & Headache & $199(3.2)$ \\
\hline 6 & Suicide attempt & $8(3.9)$ & Feeling abnormal & $189(3.1)$ \\
\hline 7 & Drug prescribing error & $7(3.4)$ & Hallucination & $174(2.8)$ \\
\hline 8 & Dizziness & $6(2.9)$ & Insomnia & $136(2.2)$ \\
\hline 9 & Middle insomnia & $6(2.9)$ & Sleep paralysis & $124(2.0)$ \\
\hline 10 & Drug administered to patient of inappropriate age & $5(2.4)$ & Adverse event & $109(1.8)$ \\
\hline
\end{tabular}

To graphically summarize areas of agreement and disagreement between Drugs.com and FAERS, we plotted the difference in rank positions of the most frequent PTs in Drugs.com from their position in FAERS for suvorexant and zolpidem (Figure 1). In this representation, a small bar indicates PTs that were ranked similarly in Drugs.com and FAERS, while a larger bar shows differing ranks between the two lists. For zolpidem, for example, sleep talking ranked as the 6th most common PT in Drugs.com, but only the 42nd most common in FAERS; thus, the rank difference is 36 . There were insufficient data from one or both sources to provide similar representations for the other three drugs studied. 
Figure 1. Difference in rank position for the 10 most common PTs in Drugs.com vs their rank in FAERS, for suvorexant and zolpidem. The rank of the Drugs.com PT was subtracted from its corresponding rank in FAERS. Bars extend to the right for PTs that held higher rank position in Drugs.com than in FAERS. For PTs that held higher rank position in FAERS compared to Drugs.com, bars extend to the left. PT: preferred term; FAERS: Food and Drug Administration Adverse Event Reporting System.

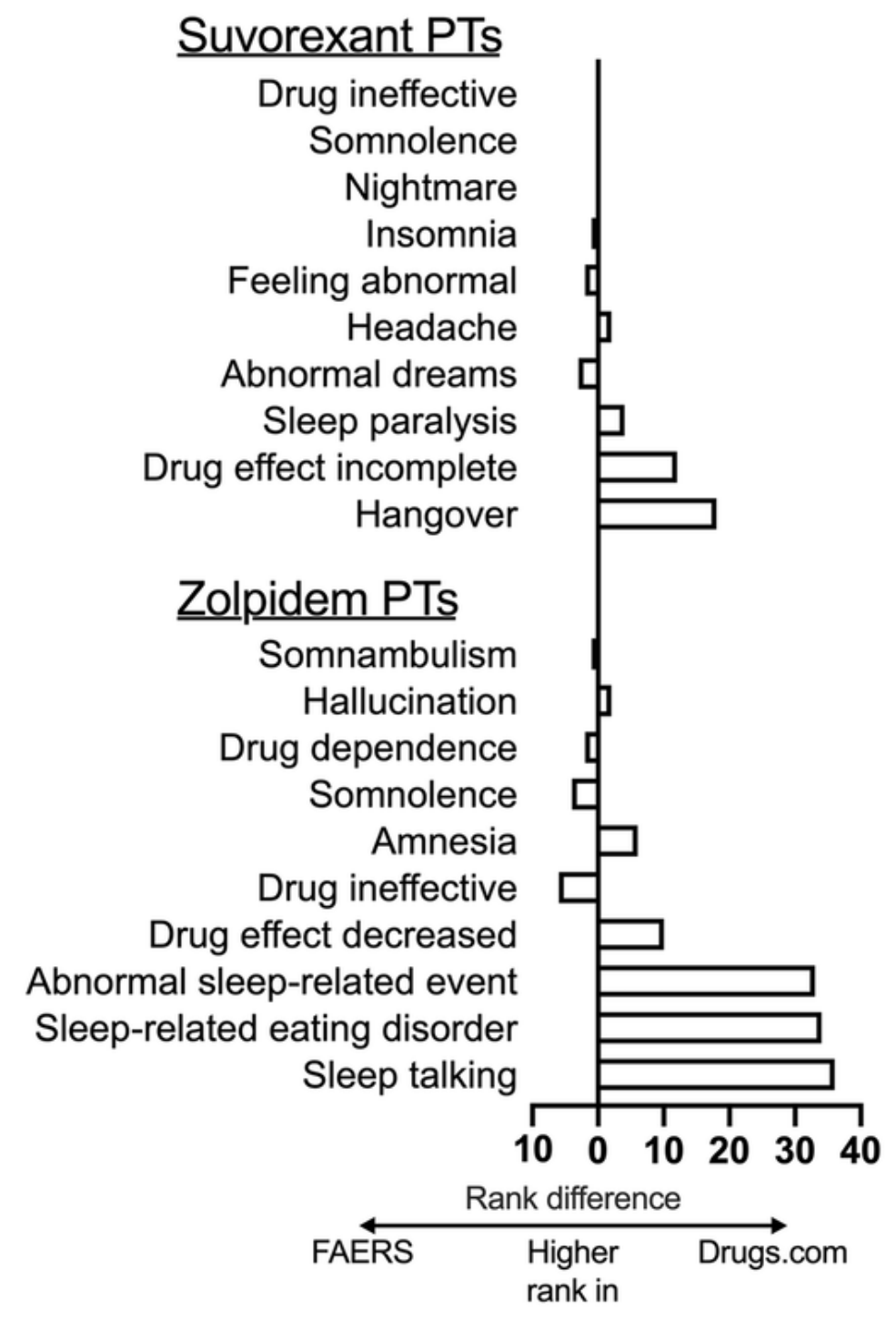

\section{Online Review User Ratings}

We next considered the Drugs.com online reviews in more detail. Figure 2A depicts the accumulation of reviews for each drug on a monthly basis for the period of February 2007 through March 2018. As of March 2018, zolpidem had the most reviews $(n=690)$, followed by suvorexant $(n=324)$, eszopiclone $(n=239)$, zaleplon $(n=82)$, and ramelteon $(n=72)$. Figure $2 B$ depicts a running monthly average of the numerical 1-10 user ratings. Zolpidem had an average rating of 7.30, followed by the other two Z-drugs, eszopiclone (6.20) and zaleplon (5.69). The nonbenzodiazepine receptor drugs ramelteon and suvorexant were rated 4.63 and 3.65 , respectively. Numerical ratings were tabulated in frequency histograms (Figure 2C), which yielded bimodal distributions for each of the five drugs. These data show that a high percentage of reviewers assigned suvorexant and ramelteon the lowest possible score of " 1 " (154/290, 53.1\% and $27 / 65,41.5 \%$, respectively). In comparison, only $10.4 \%$ $(66 / 637)$ of zolpidem reviewers rated it as "1," while $31.6 \%$
(201/637) rated it "10." Analysis of user ratings using Kruskal-Wallis test followed by the Dunn post hoc test found that zolpidem was rated significantly higher than the other four drugs, while suvorexant was rated significantly lower than all the Z-drugs (Table 5).

We then explored factors that might contribute to high or low user ratings. Focusing specifically on comments of poor efficacy, we found a statistically significant association between low user ratings and reviews that were coded for the PTs drug ineffective or drug effect incomplete for all five drugs (Figure 3 ). The odds ratios represent the increased odds of an ineffective complaint relative to a unit decrease in user rating. The number of reviews that were coded for drug ineffective or drug effect incomplete was 44/239 (18.4\%) for eszopiclone, 25/72 (35\%) for ramelteon, 165/324 (50.9\%) for suvorexant, $17 / 82(20.7 \%)$ for zaleplon, and 34/690 (4.9\%) for zolpidem. In addition, a Poisson regression analysis found a significant inverse correlation between the numerical rating and the number of distinct PTs captured from a review for all five drugs (Table 6). 
Figure 2. Statistics for consumer reviews of hypnotics on the website Drugs.com. (A) Accumulation of consumer reviews per month. Running total number of reviews are depicted for eszopiclone, ramelteon, suvorexant, zaleplon, and zolpidem. (B) Running monthly average, weighted by frequency, based on reviewers' numerical summary ranking (1-10), with 1 indicating a very poor experience and 10 indicating a very positive experience. (C) Percent frequency histograms for numerical (1-10) user ratings of insomnia drugs from drugs.com. Data are inclusive of reviews posted between February 2007 and March 2018

A

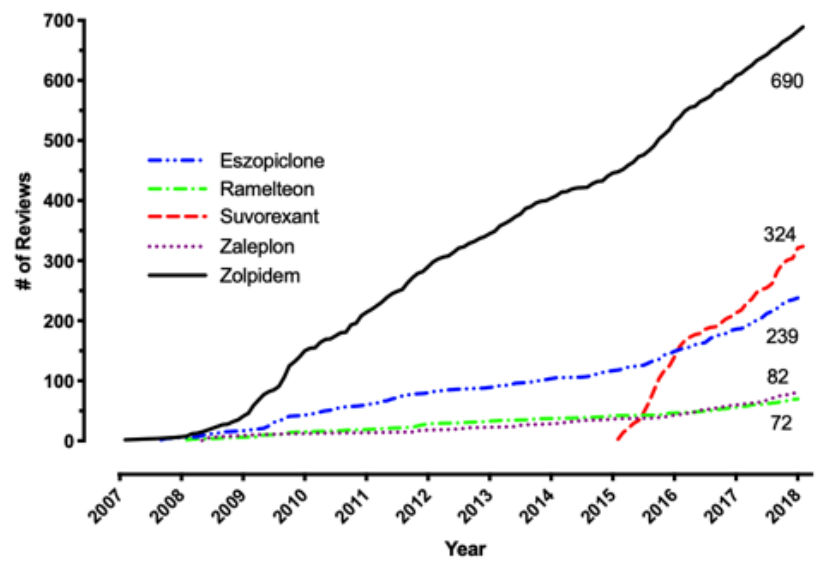

C
B

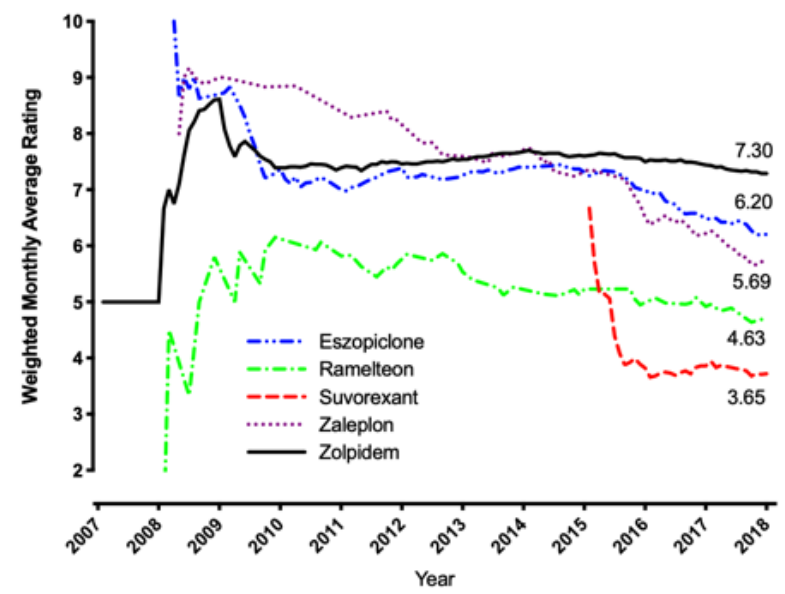

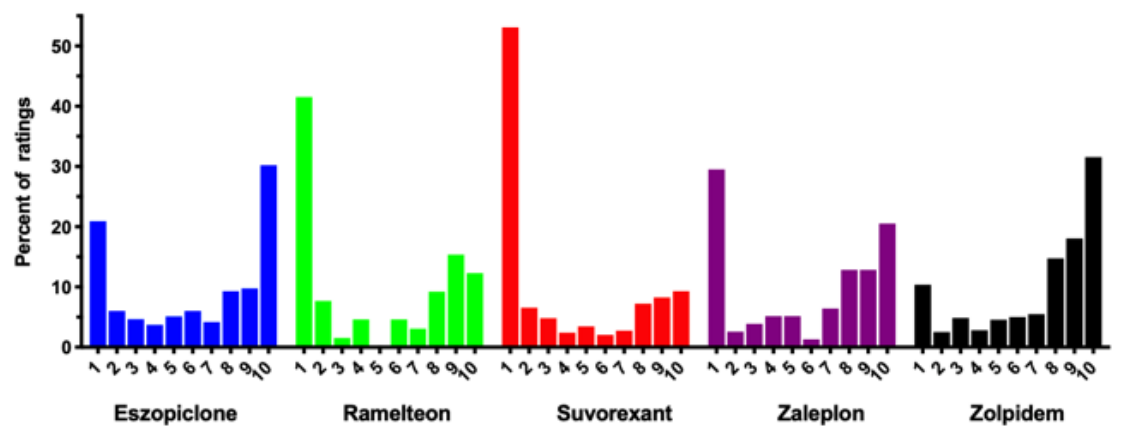

Table 5. Comparisons of hypnotic user ratings in Drugs.com with Kruskal-Wallis followed by Dunn post hoc.

\begin{tabular}{ll}
\hline Drug - comparator (median user rating) & Adjusted $P$ value \\
\hline Ramelteon (3) - eszopiclone (7) & .003 \\
Suvorexant (1) - eszopiclone (7) & $<.001$ \\
Suvorexant (1) - ramelteon (3) & .10 \\
Zaleplon (7) - eszopiclone (7) & .09 \\
Zaleplon (7) - ramelteon (3) & .11 \\
Zaleplon (7) - suvorexant (1) & $<.001$ \\
Zolpidem (8) - eszopiclone (7) & .003 \\
Zolpidem (8) - ramelteon (3) & $<.001$ \\
Zolpidem (8) - suvorexant (1) & $<.001$ \\
Zolpidem (8) - zaleplon (7) & $<.001$ \\
\hline
\end{tabular}


Figure 3. Odds ratio plot of the univariate logistic regression models to assess the relationship between user ratings and patient complaints of poor efficacy, that is, reviews that were coded for drug ineffective or drug effect incomplete. Odds ratios and $95 \%$ CIs are depicted by the circle and whiskers, respectively.

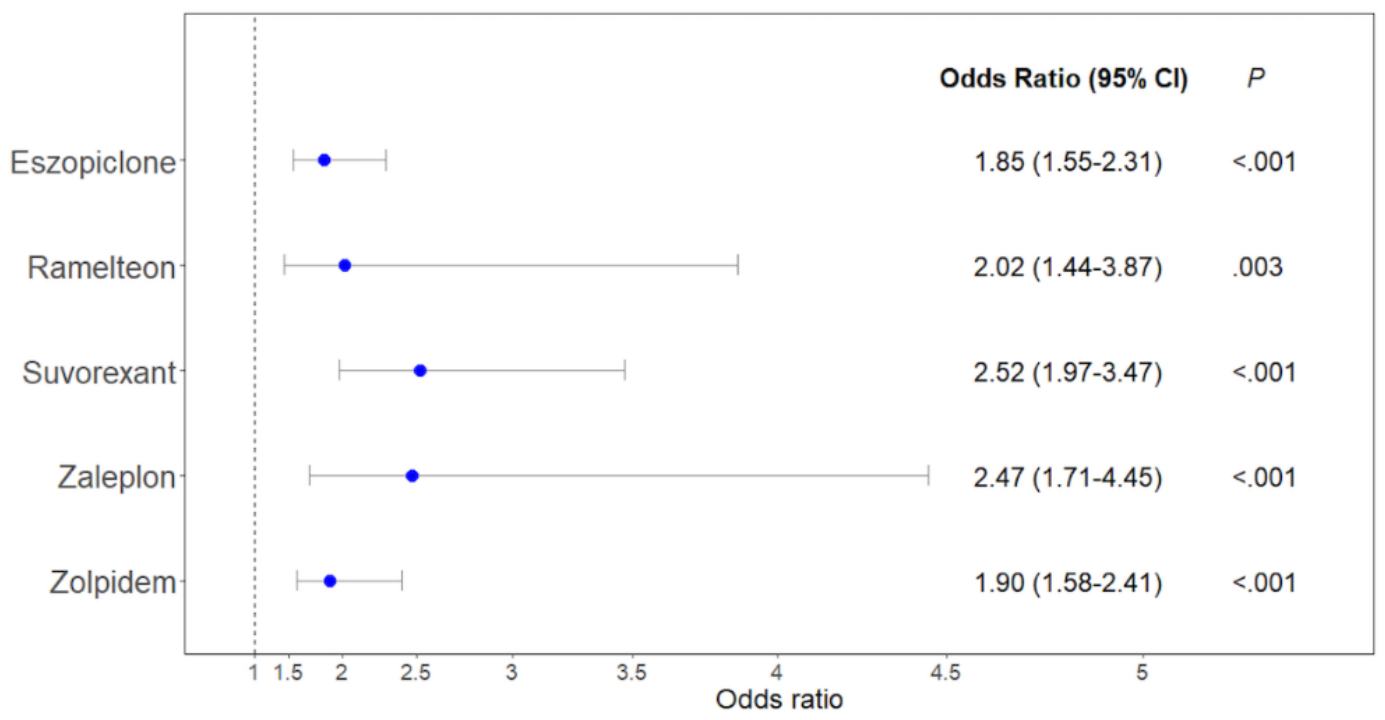

Table 6. Estimated incidence rate ratios for the effect of user ratings on the number of adverse events coded from patient reviews. The expected count of PTs is multiplied by a factor of the estimated incidence rate ratios when the user rating increases by one unit.

\begin{tabular}{lll}
\hline Drug & Incidence rate ratios $(95 \% \mathrm{CI})$ & $P$ value \\
\hline Eszopiclone & $0.89(0.85-0.92)$ & $<.001$ \\
Ramelteon & $0.90(0.83-0.97)$ & .007 \\
Suvorexant & $0.90(0.86-0.95)$ & $<.001$ \\
Zaleplon & $0.88(0.79-0.98)$ & $<.001$ \\
Zolpidem & $0.88(0.86-0.90)$ & $<.001$ \\
\hline
\end{tabular}

The estimated effect sizes correspond to a percent change in the incidence rate of PTs by $11 \%$ in eszopiclone, $10 \%$ in ramelteon, $10 \%$ in suvorexant, $12 \%$ in zaleplon, and $12 \%$ in zolpidem for a one-score decrease in the user rating. Considering eszopiclone, for example, this suggests that the expected count of PTs decreased by a factor of 0.89 when the user rating increased by one unit.

Users also mentioned cost in the reviews, most commonly with suvorexant $(75 / 324,23.1 \%)$ and eszopiclone $(37 / 239,15.5 \%)$. Univariate logistic regression showed that the tendency to mention cost did not predict lower numerical ratings for any of the drugs (Multimedia Appendix 1). For eszopiclone, however, each one-unit increase in user rating increased the odds of a comment about cost by 1.24 , suggesting that cost commentary may be correlated with a higher eszopiclone rating. Zaleplon was not included in this analysis due to insufficient data, with only 1 of 82 reviews mentioning cost.

Drugs.com does not collect demographic data for individual reviewers, but they compile top-level information on user age and country of residence, which was provided to us after sending an email request to their user support (Multimedia Appendix 2 ). These data show that more than half $(54 \%)$ of the reviews are contributed by patients aged $\leq 44$ years and that most reviewers are female (57\%) and from the United States (70\%).
We also extracted from FAERS patient demographics data on indication of therapy and reporter information, which are summarized in Multimedia Appendix 3. A plurality of reports concerned female patients $(2935 / 5916,49.6 \%)$, and the most common indications were sleep disorders (3735/5916, 63.1\%). A substantial fraction of the cases did not report gender $(1122 / 5916,19 \%)$, age $(4054 / 5916,68.5 \%)$, or indication (2102/5916, 35.5\%). Most cases (4618/5916, 78.1\%) were reported by the consumers themselves, while nearly all of the remainder were reported by health care professionals. More than 9/10 FAERS cases were contributed by reporters in the United States $(5383 / 5916,91 \%)$. Patient age was normally distributed around a mean of 56.9 (SD 18.2) years (Multimedia Appendix 4).

\section{Discussion}

\section{Principal Findings}

In general, we found that adverse event data interpreted from patient online reviews were consistent with the adverse event data in FAERS. Both resources show that poor efficacy is common with all five drugs and that amnesia is frequent with Z-drugs. A variety of adverse events particular to the individual drugs were also evident in both, for example, nightmare with suvorexant, dysgeusia and product substitution issue with eszopiclone, and dizziness with ramelteon. 
The two sources showed good agreement in zolpidem adverse events (drug ineffective, somnambulism, somnolence, drug dependence, and amnesia were among the top 10 most frequent PTs in both), but the online reviews contained a higher rate of partial sleep activities, such as walking, talking, and eating while semiconscious (for sample reviews, see Multimedia Appendix 5). Reviewers often did not remember those events, which in turn contributed to a high incidence of amnesia in the online data versus FAERS data (16.8\% vs $1.5 \%)$. This discrepancy between the two sources may be explained by the fact that the propensity for zolpidem-induced partial sleep activities is now so well known that health professionals see little value in reporting it, which would lead to an underestimation of those events in recent FAERS quarterlies. The opposite trend may be occurring in the online review data, where many reviewers seemed eager to share those stories. Such perspectives might be expected from a younger population that is more likely to participate in online activity [22,33] and appears to be more heavily represented in Drugs.com compared to FAERS. Indeed, patient age is one of the notable differences between the two resources: The average age in FAERS was nearly 60 years, while over half of the Drugs.com user population is aged $\leq 44$ years. Regardless, the high numerical ratings for zolpidem (Table 5) suggest that online reviewers may be trivializing the very serious dangers of Z-drug impairment [34], which recently prompted an FDA-mandated boxed warning for all three Z-drugs [35]. Other studies have found a general disregard of serious safety issues among the online community. For example, official safety warning announcements regarding zolpidem registered on social media in only limited and transient fashion [36], and online reviewers minimized the dangers associated with sibutramine, a weight-loss drug that was ultimately withdrawn for safety reasons [22]. A related issue was described by Adusumalli et al [24], who noted that patients may tend to rate addictive drugs more highly than alternatives that are less addictive but may be equally effective [24]. This suggests that patient opinion of zolpidem and other Z-drugs might be positively correlated with their habit-forming tendencies.

For suvorexant, we found strong agreement between the two sources. Eight adverse event PTs were among the top 10 most frequent in both sources: drug ineffective, nightmare, somnolence, headache, sleep paralysis, abnormal dreams, feeling abnormal, and insomnia. The high incidence of drug ineffective is in agreement with the reported incidence in another recent study [37], which found that suvorexant is one of the most common drugs associated with this complaint in the FAERS database. Parasomnias were also frequent, especially nightmares, which deserve special comment because they seem to be rather more intense than a typical "scary" dream, with numerous patients describing them in exceptionally vivid and terrifying themes (Multimedia Appendix 5). Here, it is important to note that nightmare was not mentioned as a potential adverse event in the suvorexant prescribing information [38] nor is it discussed in an exhaustive review of the discovery and clinical development of suvorexant [29]. This discrepancy might be explained if the premarket trials detected only mildly disturbing dreams that were coded not as nightmare but as abnormal dreams - an adverse event that is explicitly described in the suvorexant prescribing information. However, our findings indicate that conflating nightmare with abnormal dreams does not provide a true picture of the user experience with this drug. This observation adds to previous studies, which also showed that patient-contributed online data can help capture adverse events that were not described in trial data evaluated by the FDA $[39,40]$.

\section{Factors Influencing Summary Drug Rating}

For all five drugs, negative ratings significantly increased the odds of the PT drug ineffectiveness and the frequency of distinct PTs coded from the text of the corresponding review. A substantial number of online reviews for suvorexant, ramelteon, and eszopiclone explicitly mentioned cost or insurance coverage, a concern that is likely to be more prominent with reviewers in the United States compared to patients from countries with national health care programs. Unexpectedly, we found that commentary about affordability did not predict low user ratings for any of the drugs, and eszopiclone reviewers who commented about cost were actually more likely to assign a higher numerical rating. We think this seeming paradox is explained by the advent of generic formulations of eszopiclone in the United States during the review period, which led to complaints of product substitution issue from reviewers who perceived less benefit from the generic formulations (Multimedia Appendix 5). Accordingly, that PT did not appear in the eszopiclone reviews until September 2014. Around the same time, the previously stably running average began a gradual downward trend (Figure 3B).

To put the patient ratings in perspective, we considered several unrelated medications noted to cause distressing adverse events. The antipsychotic olanzapine causes significant weight gain and metabolic disorder but has a 6.7 average rating on Drugs.com [41]. Methotrexate, used frequently for rheumatoid arthritis, is associated with gastrointestinal upset, painful mouth ulcers, and fatigue, but has a rating of 7.0 [42]. The antibiotic clindamycin is rated 5.9, despite causing diarrhea in a high percentage of patients [43]. This brief sampling suggests that even drugs with onerous adverse events can earn a positive rating, as long as patients perceive real benefit. The exceedingly low ratings of suvorexant and ramelteon were even more remarkable when viewed in this context. In the larger view, our observations suggest that online patient evaluations of hypnotics are guided by fundamental considerations of drug efficacy and tolerability.

\section{Patient Perspective and Practical Utility of Online Reviews}

A patient-reported outcome is a patient's assessment of their own response to therapeutic intervention that is not reinterpreted or filtered by a clinician [44,45]. Because patients tend to evaluate their response to therapy in a holistic sense, patient-reported outcomes are useful evaluations of functional status and quality of life. These patient-reported outcomes are collected with validated instruments during drug development trials, but after a drug is marketed, there is a continued need to capture the patient's opinion in the assessment of therapeutic response. Online reviews seem well suited for recording the patient perspective, especially for drugs like hypnotics, where the balance of efficacy versus adverse events relates directly to 
the functional benefits that accrue with therapeutic success, that is, improved sleep. Furthermore, hypnotics should be reconsidered with regard to the patient's perspective because guidance on examining patient-reported outcomes [46,47] was published after trials of several important hypnotic medications were completed [48]. In addition, clinical trial participants differ from real-world patients [49]; therefore, even hypnotics with historical patient-reported outcomes can benefit from continued surveillance of the patient perspective in the form of online reviews.

In the most practical terms, online reviews can serve as an initial source of information for patients seeking a mix of views on the potential advantages and disadvantages of a given drug therapy. Compared to professional resources with similar information, online reviews are written with language and tone that resonate with the average patient [21,50]. The recently introduced FAERS dashboard can also educate consumers about drug adverse events [51]; however, interpretation of those data is still quite challenging for untrained people. Regardless of the resource, health care professionals should be ready to correct a number of common misconceptions. Patients must understand that a report of an adverse event does not establish causality, nor can individual probability of experiencing an adverse event be inferred from adverse event reports. Such a discussion can help the clinician develop an optimal plan of care that fully considers patient concerns about adverse events and expectations of effectiveness.

\section{Limitations}

The most obvious limitation of this study is the suspect validity of patient online data. We observed no reason to doubt the sincerity of these reviews, but the general vulnerability of patient reviews to misinformation and manipulation must be kept in mind, as other investigators have cautioned [52]. The subjective nature of patient self-evaluations may also be an issue, although it should be noted that self-reported data are standard for clinical sleep studies [48], which often rely on the subjects themselves to record their data and observations in a daily "sleep diary" [53].

Inherent differences in size and complexity between the two data sources made it difficult to compare the data pertaining to the same time period. We needed all the online reviews available since Drugs.com introduced this feature (in 2008); otherwise, those data sets would be too small. In contrast, even just a few years of FAERS data can become overwhelmingly large, and FAERS conventions undergo periodic changes to MedDRA coding practices and formatting of the quarterly files. In view of those complications, we opted for a simple solution that limited our FAERS survey to 11 quarters, following the approval of suvorexant in August 2014. This compromise facilitated a comparison of two sets of concurrent suvorexant data, the agent with the most limited clinical experience. Perhaps, this approach contributed to the high degree of agreement between FAERS and Drugs.com for suvorexant; if so, it probably also explains some of the disagreement for the other drugs, where the data timelines overlap less.

Other limitations concern the sparse nature of the online data compared to reports collected by regulatory agencies [54]. Although Drugs.com collects limited data on their user population, individuals rarely volunteered their age, gender, or medication dosage in the text of their reviews. Furthermore, it is often impossible to know what additional drugs they may have taken concomitantly, which could be the primary source of the adverse event. This consideration is especially relevant to these hypnotics, because all are subject to hepatic metabolism and attendant drug-drug interactions. Because follow-up clarification of case information is not possible with anonymous online testimonials lacking a verifiable patient, these limitations are fixed [55]. In contrast, we noted that many FAERS reports also lacked basic data including age, gender, and indication. Most revealing is the fact that more than three quarters of the FAERS cases $(4618 / 5916,78.1 \%)$ were reported by consumers, which means that these FAERS reports apparently share the same untrained origins as the online reviews, regardless of subsequent coding and processing by professionals.

\section{Conclusions}

Our work adds to a growing body of literature that has explored the utility of online reviews. Patient numerical ratings of hypnotic medications were influenced by perceptions of efficacy and adverse events, but not by cost. Patients rated zolpidem the highest, while ramelteon and suvorexant were held in relatively poor regard. Patient online reviews emphasized many of the same hypnotic adverse events that are reported to the FDA, most notably lack of efficacy. Future research is needed to determine how online reviews may help collect patient-reported outcomes in the postmarketing realm. Because our results show that online reviews are guided primarily by considerations of drug efficacy and tolerability and match well with adverse event data reported to FAERS, we conclude that patient online reviews offer a valuable supplement to traditional adverse event reporting systems.

\section{Acknowledgments}

We are grateful to Michelle Sloan for help with data analysis, and Ameir Barakat and Peter Lamar for proofreading. Midwestern University had no involvement in study design; collection, analysis, and interpretation of data; writing of the report; or the decision to submit the article for publication. MedDRA trademark is registered by the IFPMA on behalf of the ICH.

\section{Authors' Contributions}

KD took primary responsibility for conducting this study. All authors contributed to the conception and study design, and all authors participated in data collection, analyses, and interpretation. KD and JSB drafted the manuscript with support from LMM and ABS. All authors contributed to revisions of the manuscript and approved the final version. 


\section{Conflicts of Interest}

None declared.

\section{Multimedia Appendix 1}

Estimated odd ratios for the relationships between cost complaints and user ratings.

[PDF File (Adobe PDF File), 43 KB-Multimedia Appendix 1]

\section{Multimedia Appendix 2}

Top-level user demographics for drugs.com, as provided by drugs.com user support.

[PDF File (Adobe PDF File), 35 KB-Multimedia Appendix 2]

\section{Multimedia Appendix 3}

Demographics of 5916 patient reports for five insomnia drugs in the Food and Drug Administration Adverse Event Reporting System. Unkn/NA: Unknown or not available; Sleep dis: Sleep disorders.

[PDF File (Adobe PDF File), 37 KB-Multimedia Appendix 3]

\section{Multimedia Appendix 4}

Histogram of patient age data in Food and Drug Administration Adverse Event Reporting System reports (mean 56.9 years, SD 18.2 years; range 0-97 years). Age data were available for 31.5\% (1862/5916) of reports. Cases recorded in nonyear units were converted to years. For cases recorded in decade units, the midpoint year was used, eg, "7 DEC" was considered 65 years of age. [PDF File (Adobe PDF File), 51 KB-Multimedia Appendix 4]

\section{Multimedia Appendix 5}

Example reviews from Drugs.com for eszopiclone, suvorexant, and zolpidem.

[PDF File (Adobe PDF File), 42 KB-Multimedia Appendix 5]

\section{References}

1. Ahmad SR. Adverse drug event monitoring at the Food and Drug Administration. J Gen Intern Med 2003 Jan;18(1):57-60 [FREE Full text] [Medline: 12534765]

2. FDA: U.S. Food and Drug Administration. Individual Case Safety Reports. URL: https://www.fda.gov/industry/ fda-resources-data-standards/individual-case-safety-reports [accessed 2019-08-11]

3. Chaplin S. The Yellow Card scheme - why are GPs under-reporting? Prescriber 2006 Aug 05;17(15):18-22. [doi: 10.1002/psb.405]

4. van der Hooft CS, Sturkenboom MCJM, van Grootheest K, Kingma HJ, Stricker B. Adverse drug reaction-related hospitalisations: a nationwide study in The Netherlands. Drug Saf 2006;29(2):161-168. [doi: 10.2165/00002018-200629020-00006] [Medline: 16454543]

5. Lopez-Gonzalez E, Herdeiro MT, Figueiras A. Determinants of under-reporting of adverse drug reactions: a systematic review. Drug Saf 2009;32(1):19-31. [Medline: 19132802]

6. Sloane R, Osanlou O, Lewis D, Bollegala D, Maskell S, Pirmohamed M. Social media and pharmacovigilance: A review of the opportunities and challenges. Br J Clin Pharmacol 2015 Oct;80(4):910-920 [FREE Full text] [doi: 10.1111/bcp.12717] [Medline: 26147850]

7. Sarker A, Ginn R, Nikfarjam A, O'Connor K, Smith K, Jayaraman S, et al. Utilizing social media data for pharmacovigilance: A review. J Biomed Inform 2015 Apr;54:202-212 [FREE Full text] [doi: 10.1016/j.jbi.2015.02.004] [Medline: 25720841]

8. Omar I, Harris E. The Use of Social Media in ADR Monitoring and Reporting. J Pharmacovigilance 2016;04(06). [doi: 10.4172/2329-6887.1000223]

9. Lardon J, Abdellaoui R, Bellet F, Asfari H, Souvignet J, Texier N, et al. Adverse Drug Reaction Identification and Extraction in Social Media: A Scoping Review. J Med Internet Res 2015 Jul 10;17(7):e171 [FREE Full text] [doi: 10.2196/jmir.4304] [Medline: 26163365]

10. Khare R, Good BM, Leaman R, Su AI, Lu Z. Crowdsourcing in biomedicine: challenges and opportunities. Brief Bioinform 2016 Jan;17(1):23-32. [doi: 10.1093/bib/bbv021] [Medline: 25888696]

11. White RW, Tatonetti NP, Shah NH, Altman RB, Horvitz E. Web-scale pharmacovigilance: listening to signals from the crowd. J Am Med Inform Assoc 2013 May 1;20(3):404-408 [FREE Full text] [doi: 10.1136/amiajnl-2012-001482] [Medline: 23467469] 
12. Pierce CE, Bouri K, Pamer C, Proestel S, Rodriguez HW, Van Le H, et al. Evaluation of Facebook and Twitter Monitoring to Detect Safety Signals for Medical Products: An Analysis of Recent FDA Safety Alerts. Drug Saf 2017 Apr;40(4):317-331 [FREE Full text] [doi: 10.1007/s40264-016-0491-0] [Medline: 28044249]

13. Comfort S, Perera S, Hudson Z, Dorrell D, Meireis S, Nagarajan M, et al. Sorting Through the Safety Data Haystack: Using Machine Learning to Identify Individual Case Safety Reports in Social-Digital Media. Drug Saf 2018 Jun;41(6):579-590 [FREE Full text] [doi: 10.1007/s40264-018-0641-7] [Medline: 29446035]

14. Bollegala D, Maskell S, Sloane R, Hajne J, Pirmohamed M. Causality Patterns for Detecting Adverse Drug Reactions From Social Media: Text Mining Approach. JMIR Public Health Surveill 2018 May 09;4(2):e51 [FREE Full text] [doi: 10.2196/publichealth.8214] [Medline: 29743155]

15. Smith K, Golder S, Sarker A, Loke Y, O'Connor K, Gonzalez-Hernandez G. Methods to Compare Adverse Events in Twitter to FAERS, Drug Information Databases, and Systematic Reviews: Proof of Concept with Adalimumab. Drug Saf 2018 Dec;41(12):1397-1410 [FREE Full text] [doi: 10.1007/s40264-018-0707-6] [Medline: 30167992]

16. Freifeld CC, Brownstein JS, Menone CM, Bao W, Filice R, Kass-Hout T, et al. Digital drug safety surveillance: monitoring pharmaceutical products in twitter. Drug Saf 2014 May;37(5):343-350 [FREE Full text] [doi: 10.1007/s40264-014-0155-x] [Medline: 24777653]

17. Matsuda S, Aoki K, Tomizawa S, Sone M, Tanaka R, Kuriki H, et al. Analysis of Patient Narratives in Disease Blogs on the Internet: An Exploratory Study of Social Pharmacovigilance. JMIR Public Health Surveill 2017 Feb 24;3(1):e10 [FREE Full text] [doi: 10.2196/publichealth.6872] [Medline: 28235749]

18. Chen JH, Altman RB. Data-Mining Electronic Medical Records for Clinical Order Recommendations: Wisdom of the Crowd or Tyranny of the Mob? AMIA Jt Summits Transl Sci Proc 2015;2015:435-439 [FREE Full text] [Medline: 26306281]

19. Eysenbach G. Infodemiology and infoveillance: framework for an emerging set of public health informatics methods to analyze search, communication and publication behavior on the Internet. J Med Internet Res 2009;11(1):e11 [FREE Full text] [doi: 10.2196/jmir.1157] [Medline: 19329408]

20. Hughes S, Lacasse J, Fuller RR, Spaulding-Givens J. Adverse effects and treatment satisfaction among online users of four antidepressants. Psychiatry Res 2017 Sep;255:78-86. [doi: 10.1016/j.psychres.2017.05.021] [Medline: 28531820]

21. Moncrieff J, Cohen D, Mason JP. The subjective experience of taking antipsychotic medication: a content analysis of Internet data. Acta Psychiatr Scand 2009 Aug;120(2):102-111. [doi: 10.1111/j.1600-0447.2009.01356.x] [Medline: 19222405]

22. Duh MS, Cremieux P, Audenrode MV, Vekeman F, Karner P, Zhang H, et al. Can social media data lead to earlier detection of drug-related adverse events? Pharmacoepidemiol Drug Saf 2016 Dec;25(12):1425-1433 [FREE Full text] [doi: 10.1002/pds.4090] [Medline: 27601271]

23. Swogger MT, Hart E, Erowid F, Erowid E, Trabold N, Yee K, et al. Experiences of Kratom Users: A Qualitative Analysis. J Psychoactive Drugs 2015;47(5):360-367. [doi: 10.1080/02791072.2015.1096434] [Medline: 26595229]

24. Adusumalli S, Lee H, Hoi Q, Koo S, Tan IB, Ng PC. Assessment of Web-Based Consumer Reviews as a Resource for Drug Performance. J Med Internet Res 2015 Aug 28;17(8):e211 [FREE Full text] [doi: 10.2196/jmir.4396] [Medline: 26319108]

25. Buysse DJ. Insomnia. JAMA 2013 Feb 20;309(7):706-716 [FREE Full text] [doi: 10.1001/jama.2013.193] [Medline: 23423416]

26. Bertisch SM, Herzig SJ, Winkelman JW, Buettner C. National use of prescription medications for insomnia: NHANES 1999-2010. Sleep 2014 Feb 01;37(2):343-349 [FREE Full text] [doi: 10.5665/sleep.3410] [Medline: 24497662]

27. Wilt TJ, MacDonald R, Brasure M, Olson CM, Carlyle M, Fuchs E, et al. Pharmacologic Treatment of Insomnia Disorder: An Evidence Report for a Clinical Practice Guideline by the American College of Physicians. Ann Intern Med 2016 Jul 19;165(2):103-112. [doi: 10.7326/M15-1781] [Medline: 27136278]

28. Kuriyama A, Honda M, Hayashino Y. Ramelteon for the treatment of insomnia in adults: a systematic review and meta-analysis. Sleep Med 2014 Apr;15(4):385-392. [doi: 10.1016/j.sleep.2013.11.788] [Medline: 24656909]

29. Coleman PJ, Gotter AL, Herring WJ, Winrow CJ, Renger JJ. The Discovery of Suvorexant, the First Orexin Receptor Drug for Insomnia. Annu Rev Pharmacol Toxicol 2017 Jan 06;57:509-533. [doi: 10.1146/annurev-pharmtox-010716-104837] [Medline: 27860547]

30. Drugs.com. Review drug. URL: https://www.drugs.com/members comments add/ [accessed 2019-01-15]

31. MedDRA. 2015. Introductory Guide MedDRA Version 18.0. URL: https://www.meddra.org/sites/default/files/guidance/ file/intguide 180 english.pdf [accessed 2019-10-30]

32. FDA: U.S. Food and Drug Administration. 2018. FDA Adverse Event Reporting System (FAERS): Latest Quarterly Data Files. URL: https://www.fda.gov/drugs/guidancecomplianceregulatoryinformation/surveillance/adversedrugeffects/ ucm082193.htm [accessed 2019-01-09]

33. Price J. What Can Big Data Offer the Pharmacovigilance of Orphan Drugs? Clin Ther 2016 Dec;38(12):2533-2545. [doi: 10.1016/j.clinthera.2016.11.009] [Medline: 27914633]

34. Gunja N. In the Zzz zone: the effects of Z-drugs on human performance and driving. J Med Toxicol 2013 Jun;9(2):163-171 [FREE Full text] [doi: 10.1007/s13181-013-0294-y] [Medline: 23456542] 
35. FDA: U.S. Food and Drug Administration. 2019. FDA adds Boxed Warning for risk of serious injuries caused by sleepwalking with certain prescription insomnia medicines. URL: https://www.fda.gov/drugs/drug-safety-and-availability/ fda-adds-boxed-warning-risk-serious-injuries-caused-sleepwalking-certain-prescription-insomnia [accessed 2019-08-11]

36. Sinha MS, Freifeld CC, Brownstein JS, Donneyong MM, Rausch P, Lappin BM, et al. Social Media Impact of the Food and Drug Administration's Drug Safety Communication Messaging About Zolpidem: Mixed-Methods Analysis. JMIR Public Health Surveill 2018 Jan 05;4(1):e1 [FREE Full text] [doi: 10.2196/publichealth.7823] [Medline: 29305342]

37. Misu T, Kortepeter CM, Muñoz MA, Wu E, Dal Pan GJ. An Evaluation of "Drug Ineffective" Postmarketing Reports in Drug Safety Surveillance. Drugs Real World Outcomes 2018 Jun;5(2):91-99 [FREE Full text] [doi: 10.1007/s40801-018-0131-3] [Medline: 29476420]

38. FDA.gov. Highlights of Prescribing Information. URL: https://www.accessdata.fda.gov/drugsatfda_docs/label/2014/ 204569s000lbledt.pdf [accessed 2019-01-09]

39. White RW, Harpaz R, Shah NH, DuMouchel W, Horvitz E. Toward enhanced pharmacovigilance using patient-generated data on the internet. Clin Pharmacol Ther 2014 Aug;96(2):239-246 [FREE Full text] [doi: 10.1038/clpt.2014.77] [Medline: 24713590]

40. Yom-Tov E, Gabrilovich E. Postmarket drug surveillance without trial costs: discovery of adverse drug reactions through large-scale analysis of web search queries. J Med Internet Res 2013;15(6):e124 [FREE Full text] [doi: 10.2196/jmir.2614] [Medline: 23778053]

41. Drugs.com. User Reviews for Olanzapine. URL: https://www.drugs.com/comments/olanzapine/ [accessed 2019-01-09]

42. Drugs.com. User Reviews for Methotrexate. URL: https://www.drugs.com/comments/methotrexate/ [accessed 2019-01-09]

43. Drugs.com. User Reviews for Clindamycin. URL: https://www.drugs.com/comments/clindamycin/ [accessed 2019-01-09]

44. Weldring T, Smith SMS. Patient-Reported Outcomes (PROs) and Patient-Reported Outcome Measures (PROMs). Health Serv Insights 2013;6:61-68 [FREE Full text] [doi: 10.4137/HSI.S11093] [Medline: 25114561]

45. Acquadro C, Berzon R, Dubois D, Leidy NK, Marquis P, Revicki D, et al. Incorporating the patient's perspective into drug development and communication: an ad hoc task force report of the Patient-Reported Outcomes (PRO) Harmonization Group meeting at the Food and Drug Administration, February 16, 2001. Value Health 2003;6(5):522-531 [FREE Full text] [doi: $10.1046 /$ j.1524-4733.2003.65309.x] [Medline: $\underline{14627058}$ ]

46. Calvert M, Blazeby J, Altman DG, Revicki DA, Moher D, Brundage MD, CONSORT PRO Group. Reporting of patient-reported outcomes in randomized trials: the CONSORT PRO extension. JAMA 2013 Feb 27;309(8):814-822. [doi: 10.1001/jama.2013.879] [Medline: 23443445]

47. Basch E. The missing voice of patients in drug-safety reporting. N Engl J Med 2010 Mar 11;362(10):865-869 [FREE Full text] [doi: 10.1056/NEJMp0911494] [Medline: 20220181]

48. Buysse DJ, Yu L, Moul DE, Germain A, Stover A, Dodds NE, et al. Development and validation of patient-reported outcome measures for sleep disturbance and sleep-related impairments. Sleep 2010 Jun;33(6):781-792 [FREE Full text] [Medline: 20550019]

49. Roehrs T, Verster JC, Koshorek G, Withrow D, Roth T. How representative are insomnia clinical trials? Sleep Med 2018 Nov;51:118-123 [FREE Full text] [doi: 10.1016/j.sleep.2018.06.003] [Medline: $\underline{\text { 30144651] }}$

50. Hughes $\mathrm{S}$, Cohen D. Can online consumers contribute to drug knowledge? A mixed-methods comparison of consumer-generated and professionally controlled psychotropic medication information on the internet. J Med Internet Res 2011;13(3):e53 [FREE Full text] [doi: 10.2196/jmir.1716] [Medline: 21807607]

51. Kumar A. The Newly Available FAERS Public Dashboard: Implications for Health Care Professionals. Hosp Pharm 2019 Apr;54(2):75-77. [doi: 10.1177/0018578718795271] [Medline: $\underline{\text { 30923396] }}$

52. Chew SW, Khoo CS. Comparison of drug information on consumer drug review sites versus authoritative health information websites. J Assn Inf Sci Tec 2015 Feb 13;67(2):333-349. [doi: 10.1002/asi.23390]

53. Carney CE, Buysse DJ, Ancoli-Israel S, Edinger JD, Krystal AD, Lichstein KL, et al. The consensus sleep diary: standardizing prospective sleep self-monitoring. Sleep 2012 Feb 01;35(2):287-302 [FREE Full text] [doi: 10.5665/sleep.1642] [Medline: 22294820]

54. Karapetiantz P, Bellet F, Audeh B, Lardon J, Leprovost D, Aboukhamis R, et al. Descriptions of Adverse Drug Reactions Are Less Informative in Forums Than in the French Pharmacovigilance Database but Provide More Unexpected Reactions. Front Pharmacol 2018;9:439. [doi: 10.3389/fphar.2018.00439] [Medline: 29765326]

55. Coloma PM, Becker B, Sturkenboom MCJM, van Mulligen EM, Kors JA. Evaluating Social Media Networks in Medicines Safety Surveillance: Two Case Studies. Drug Saf 2015 Oct;38(10):921-930 [FREE Full text] [doi:

10.1007/s40264-015-0333-5] [Medline: 26242616]

\section{Abbreviations}

FAERS: Food and Drug Administration Adverse Event Reporting System

LLT: low-level term

MedDRA: Medical Dictionary for Regulatory Activities

PT: preferred term 


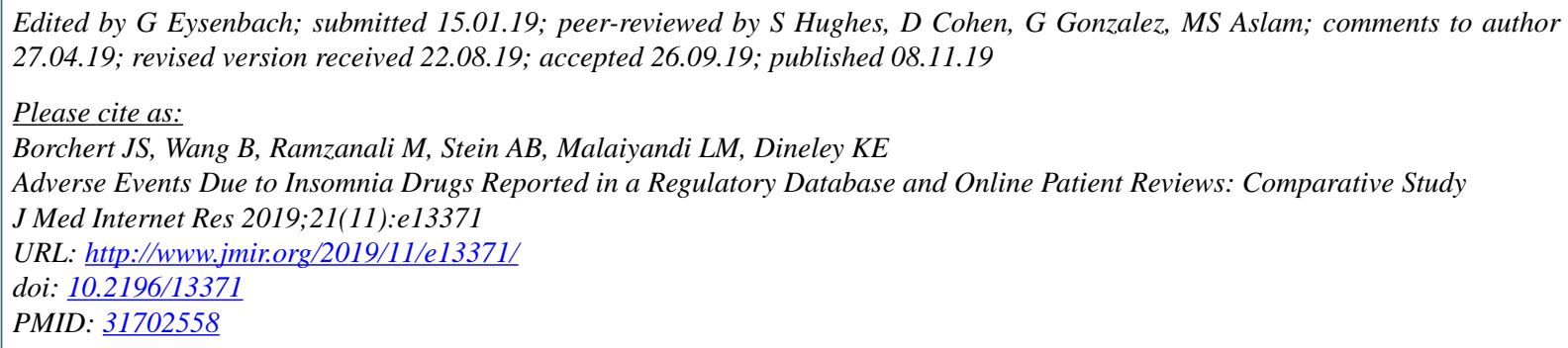

(CJill S Borchert, Bo Wang, Muzaina Ramzanali, Amy B Stein, Latha M Malaiyandi, Kirk E Dineley. Originally published in the Journal of Medical Internet Research (http://www.jmir.org), 08.11.2019. This is an open-access article distributed under the terms of the Creative Commons Attribution License (https://creativecommons.org/licenses/by/4.0/), which permits unrestricted use, distribution, and reproduction in any medium, provided the original work, first published in the Journal of Medical Internet Research, is properly cited. The complete bibliographic information, a link to the original publication on http://www.jmir.org/, as well as this copyright and license information must be included. 